Running Head: ANXIETY, CONTEXT AND PERCEPTUAL-MOTOR SKILL

The effects of anxiety and situation-specific context on perceptual-motor skill: A multi-level investigation

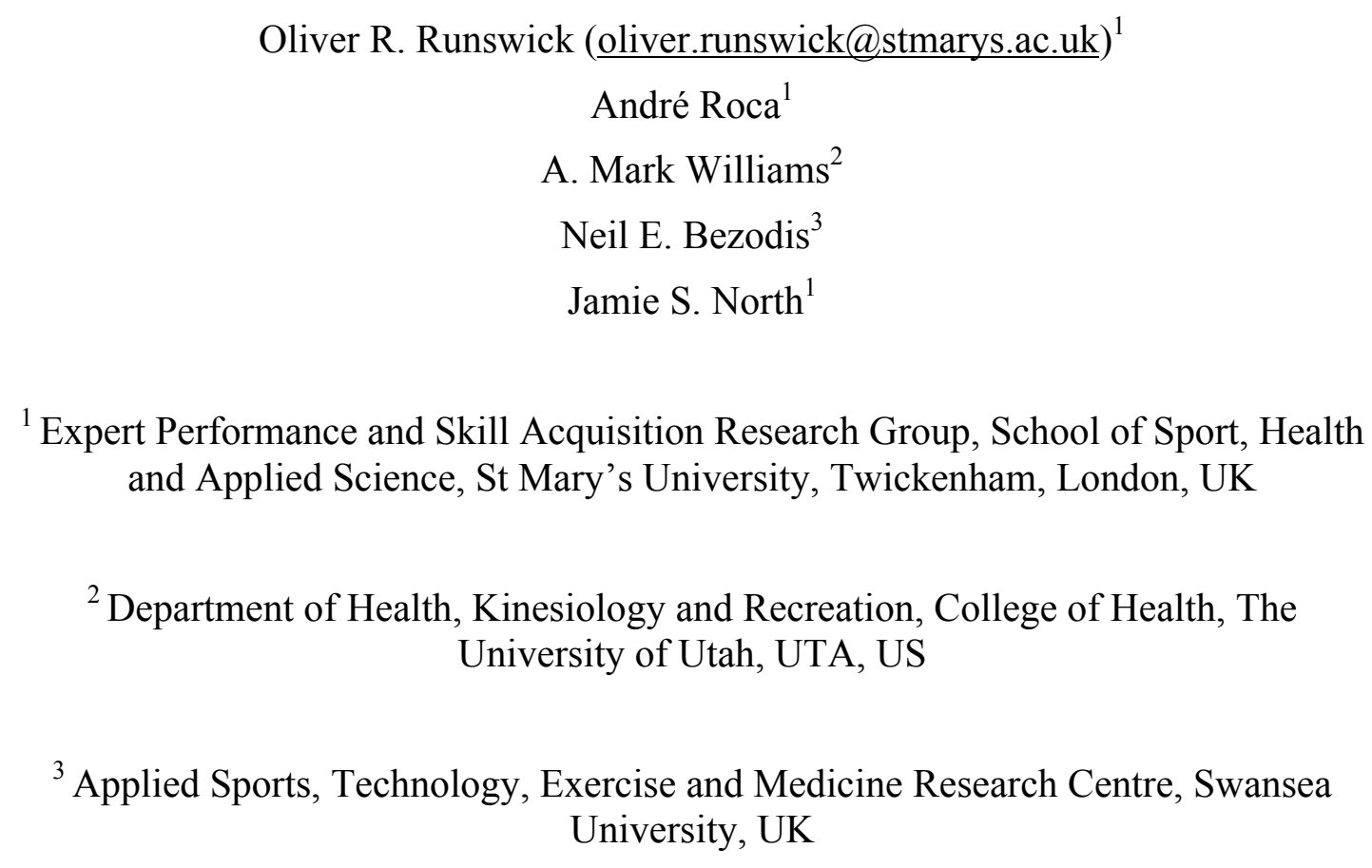

${ }^{1}$ Expert Performance and Skill Acquisition Research Group, School of Sport, Health and Applied Science, St Mary's University, Twickenham, London, UK

${ }^{2}$ Department of Health, Kinesiology and Recreation, College of Health, The University of Utah, UTA, US
${ }^{3}$ Applied Sports, Technology, Exercise and Medicine Research Centre, Swansea University, UK Accepted for publication in Psychological Research on 04/03/2017

24 Corresponding author:

25 Oliver Runswick

6 School of Sport, Health and Applied Science

27 St Mary's University

28 Waldegrave Road

29 Twickenham

London

31 United Kingdom

2 TW1 4SX

33 Email: oliver.runswick@stmarys.ac.uk

34 Phone: +44 (0)20 82408243 
2 We examined the effects of anxiety and situation-specific contextual information on

3 attentional, interpretational, and behavioural processes underpinning perceptual-motor

4 performance as proposed by Nieuwenhuys and Oudejans (2012) using an in-situ task.

5 Twelve skilled cricket batsmen played against a skilled spin bowler under conditions

6 manipulated to induce low- and high-levels of anxiety and the presence of low- and

7 high-levels of situation-specific context. High anxiety decreased the number of good

8 bat-ball contacts, while high levels of situation-specific context increased the number

9 of times the ball was missed. When under high anxiety, participants employed

10 significantly more fixations of shorter duration to more locations, but the effects of

11 anxiety were restricted to the attentional level only. Situation-specific context affected

12 performance and behavioural measures but not anxiety, cognitive load or perceptual-

13 cognitive processes, suggesting that performance is influenced through different

14 mechanisms from anxiety that are independent of working memory load.

17 Key Words: perceptual-cognitive expertise; emotion; working memory; cognitive

18 load 


\section{The effects of anxiety and situation-specific context on perceptual-motor skill: A}

\section{multi-level investigation}

Anxiety is defined as an aversive emotional state that can occur in threatening situations and increase thoughts of worry and self-doubt (Derakshan \& Eysenck, 2009) often leading to a decrement in performance. This negative relationship between anxiety and performance has attracted considerable attention in the literature (e.g., Eysenck, Derakshan, Santos, \& Calvo, 2007; Janelle, 2002). In their Integrated Model of Anxiety and Perceptual Motor Performance, Nieuwenhuys and Oudejans (2012) reported three operational levels at which anxiety can influence goal-directed actions, namely, attentional, interpretational, and behavioural. They suggested that performance under anxiety is primarily affected by the limited capacity of working memory and that anxiety can cause an increase in the use of stimulus driven attentional processes that respond to salient or conspicuous stimuli (see Corbetta \& Shulman, 2002). An increase in the use of these bottom-up stimulus driven processes will, in turn, lead to threat-related attention, interpretation, and response tendencies. Nieuwenhuys and Oudejans's (2012) model is grounded in Attentional Control Theory (ACT; Eysenck, Derakshan, Santos, \& Calvo, 2007) and its predecessor Processing Efficiency Theory (PET; Eysenck \& Calvo, 1992). We tested the predictions of ACT by investigating the effects of anxiety on processing efficiency and performance effectiveness. We also tested the predictions of Nieuwenhuys and Oudejans's (2012) integrated model of anxiety and perceptual motor performance by examining how manipulations of situation-specific context and anxiety using a novel in-situ task affected perceptual-motor performance at attentional, interpretational, and behavioural levels. 
The PET (Eysenck \& Calvo, 1992) distinguishes between processing

2 efficiency and performance effectiveness; performance effectiveness refers to the

3 quality of performance produced, while efficiency refers to the amount of resources

4 required to produce that level of performance. The PET predicts that worrisome

5 thought consumes some of the limited resources of working memory, leaving fewer

6 resources available for execution of the task. The main effects of worry limiting

7 resources are focused on the central executive and it is assumed that tasks which place

8 significant demands on this system will suffer to a greater extent under increased

9 anxiety.

Eysenck et al. (2007) identified several limitations concerning a lack of

precision in the predictions of PET and proposed Attentional Control Theory (ACT)

to address these issues. ACT builds on the foundation set by PET and presents six

hypotheses relating to the effects anxiety has on specific functions of the central

executive and, subsequently, on processing efficiency and performance effectiveness.

These effects relate to changes in cognitive load and specific functions of the central executive, namely shifting, inhibition, and updating (Miyake, Friedman, Emerson,

Witzki, Howerter, \& Wager, 2000). Shifting refers to one's ability to shift attention

between multiple concurrent tasks, which ACT predicts will be affected negatively by

the presence of anxiety. Inhibition refers to one's ability to deliberately avoid

distraction by task-irrelevant stimuli. The final central executive function refers to the updating and monitoring of working memory representations (i.e., representations produced from the phonological loop and visuo-spatial sketchpad). It is suggested that performance of an individual can be maintained under anxiety by increasing the use of working memory resources to execute the functions of the central executive, albeit reducing processing efficiency. If one reaches the point where no further working 
1 2 effectiveness can be adversely affected. anxiety and offer support for ACT.

memory resources are available to counter the effects of anxiety, performance

Visual search behaviour has frequently been used as an indicator of visual attention and processing efficiency, with researchers showing clear changes in gaze characteristics as a function of anxiety (e.g., Wilson, Smith, \& Holmes, 2007; Wilson, Vine, \& Wood, 2009). Typically, in closed skills, such as a soccer penalty kick or cricket batting, as people become more anxious they show less efficient visual search behaviours by making more fixations of a shorter duration, and are more easily distracted by irrelevant stimuli (for a review, see Janelle, 2002). Wilson et al. (2009) found that in a soccer penalty kick task, when participants were anxious their attempts became less accurate, while their first visual fixation became shorter and they spent more time fixating on threat-related stimuli (i.e., the goalkeeper). Similarly, Causer, Holmes, Smith, and Williams (2011) revealed that elite shotgun shooters made final fixations of a shorter duration and shot less accurately when performing under anxiety in comparison to no-anxiety conditions. These changes in visual search strategy show how attentional and perceptual processes (and in turn performance) are affected by

When testing ACT and PET, researchers have commonly employed protocols that involve abstract secondary tasks or non-task relevant stimuli to load working memory and test shifting and inhibition functions. For example, Murray and Janelle (2003) used a dual-task paradigm in which participants completed the primary task of racing in a driving simulator while being assessed on a secondary task which required them to respond quickly and accurately to a light stimulus that appeared at random in the centre or periphery of the display. However, researchers are increasingly highlighting the importance of ensuring that experimental environments faithfully represent the 
1 performance environment to which the results will be generalised (see Broadbent,

2 Causer, Williams, \& Ford, 2014; Dicks, Button, \& Davids, 2010; Pinder, Davids,

3 Renshaw, \& Araújo, 2011; Stone, Panchuk, Davids, North, \& Maynard, 2014).

$4 \quad$ It has been suggested that representative task designs could help bridge the gap

5 between research and application in the field (Broadbent et al., 2014). Muller,

6 Brenton, and Rosalie (2015) discussed methodological considerations specific to

7 investigating expert interceptive skill in in-situ settings and suggested the more

8 representative the design the easier it is to generalise findings to the visuomotor

9 responses that would occur in real-world settings. Furthermore, Cañal-Bruland and

10 Mann (2015) argued that when examining anticipation and other perceptual-cognitive

11 skills, greater attention must be given to the role of probabilistic or contextual

12 information. Researchers investigating representative task design have typically

13 focused solely on ensuring perceptual information is faithfully represented (see Dicks

14 et al., 2010; Pinder et al., 2011a; Stone et al., 2015) and few have examined how

15 information such as score-line, time of the match or positioning of opponents provides

16 important context that is more cognitive in nature and may affect working memory

17 load. Just as it is important to accurately represent perceptual information, so too is it

18 vital to ensure that working memory and cognitive load are targeted using

representative context-specific manipulations rather than abstract secondary tasks in

order to investigate how this affects perceptual-motor performance and interacts with anxiety.

The Oxford Dictionaries (2016) define context as "the circumstances which form

the setting for an event, statement or idea, and in terms of which it can be fully

understood." In the scientific literature, this definition has been applied in several 
1 game and batters on base in baseball, while McRobert, Ward, Eccles, and Williams

2 (2011) used the sequenceing of deliveries by bowlers in cricket to manipulate context.

3 Both found that more contextual information improved the players' ability to predict

4 the nature of the pitch/delivery. Cocks, Jackson, Bishop, and Williams (2015)

5 combined context with anxiety to investigate ACT and how anxiety can affect the use

6 of high- and low-order cognitive processes. They manipulated context through

7 postural information, shot sequencing, and player positioning in a tennis simulation

8 and found anxiety was most detrimental to performance in conditions where only

9 contextual information was given and stimulus driven information was omitted.

10 Critically, Cocks et al. (2015), McRobert et al. (2011), and Paull and Glencross

11 (1997) tested effects of context on anticipation performance with no motor

12 performance or behavioural measures. Although such methods allow for insight into

13 perceptual and cognitive processes, the omission of the motor element makes these

14 findings difficult to translate to performance environments.

In this paper, we differentiate between situation-specific and non situation-specific context. Situation-specific context refers to information leading to the selection and performance of a skill that is unique to that event. In cricket batting the time left in the game, score, position of opposition fielders, and sequencing information from a bowler are unique to each time the ball is bowled. Non-situation specific context is not unique to a single event and includes contextual information that can be found at any time during performance. For example, information about past team successes and playing style is independent from the situation of the game. Situation-specific context can be more easily manipulated in training and research environments and is likely to provide new information that needs to be processed and therefore increase working memory load. 
Although researchers have largely concentrated on examining how anxiety and working memory load affect perceptual and attentional processes, some researchers

3 have shown how anxiety effects can emerge at cognitive decision-making levels.

$4 \quad$ Pijpers, Oudejans, Bakker, and Beek (2006) used a traversing task on a climbing wall

5 at high and low distances from the ground and found a decrease in perceived reaching

6 ability under anxiety (higher traverse). Nieuwenhuys, Savelsbergh, and Oudejans

7 (2012) investigated the link between perceptual behaviour and decision making in a

8 task requiring police officers to choose between "shoot" and "don't shoot" options in

9 a training task. Anxiety was manipulated with the use of a 'shoot back cannon' that returned fire at participants using small plastic bullets. No changes were found in perceptual behaviour under anxiety or between correct and incorrect responses, however, incorrect decisions were shown to be $20 \%$ quicker than correct decisions, suggesting that even without a change in perceptual behaviour, officers were more inclined to take decisions quickly under anxiety leading to more wrong decisions.

These studies suggest that anxiety can cause a reduction in response options and an increase in the speed of decision making at the interpretational level.

The third level identified in Nieuwenhuys and Oudejans's (2012) model is behavioural (i.e., the technical execution of movement responses), which has also been shown to be affected under conditions of heightened anxiety. In the study by Nibbeling, Daanen, Gerritsma, Hoifland, and Oudejans (2012), where runners were asked to run on a treadmill placed high above the ground, not only did they report cognitive thoughts of falling but they also ran with shorter steps and an increased step 23 frequency and ground contact time compared with running on a treadmill low to the ground. No researchers have investigated all three levels simultaneously using an insitu task. 
In the current paper, we examine how situation-specific (i.e., representative)

2 contextual information and anxiety affect perceptual-motor skill and the mechanisms

3 employed at attentional (perceptual), interpretational (cognitive), and behavioural

4 (motor) operational levels using an in-situ task. We employed a situation-specific

5 context manipulation to alter working memory load and test ACT using a

6 representative task design. We hypothesised that under high anxiety, participants

7 would make more fixations of shorter duration to more locations of less relevance

8 and, based on the hypothesis emanating from ACT, that processing efficiency will be

9 affected to a greater extent than performance effectiveness. Using Nieuwenhuys and

10 Oudejans's model (2012), we hypothesised that as well as decreased efficiency of

11 attentional behaviour, anxiety will negatively affect the number of response options

12 available at the interpretational level and lead to a decrement in the quality of batting

13 movement execution and performance. Under context-laden conditions, we

14 hypothesised that when coupled with high anxiety there will be insufficient resources

15 available to compensate for the effects of anxiety, negatively affecting performance.

16 At attentional and interpretational levels, we expected the effects to be in line with

17 McRobert et al. (2011) who showed a decrease in the efficiency of attentional

18 mechanisms and an increase in thought processes relating to evaluation and planning

19 under increased context. We predicted that, similar to anxiety, there would be a

20 consequent effect of changes at attentional and interpretational levels causing a

21 decrease in efficiency at the behavioural level. Finally, according to ACT, both

22 anxiety and context manipulations will increase load on working memory, and

23 therefore we hypothesised the negative effects on performance to be additive and that

24 no interaction will be reported. 


\section{Participants}

3 Twelve skilled cricket batsmen $(M$ age $=22.2 \pm 3.4$ years $)$ played against a skilled

$4 \quad$ spin bowler. Participants were experienced players $(M=14.3 \pm 4.7$ years $)$ playing at

5 senior amateur club level with five individuals having played at a regional level. The

6 bowler was 23 years old and had experience playing County level cricket and was

7 currently playing at club and minor counties level. Participants spent between six and

820 hours a week playing cricket $(M=13.5 \pm 5.2$ hours $)$. Procedures conformed to the

9 ethical standards of the Declaration of Helsinki. Ethical approval was granted and the

10 research was conducted in accordance with the ethical guidelines of the lead

11 university. Written informed consent was obtained from all individual participants.

\section{Inventories and Apparatus}

The Mental Readiness Form-Likert (MRF-L). The MRF-L (Krane, 1994)

was used as a measure to assess cognitive anxiety, somatic anxiety, and selfconfidence with participants responding on a Likert scale from one to eleven. The cognitive anxiety scale rates thoughts $(1=$ calm, $11=$ worried $)$, the somatic anxiety scale rates how the body feels $(1=$ relaxed, $11=$ tense $)$, and the confidence scale rates feeling $(1=$ confident, $11=$ scared $)$. Krane (1994) reported moderate to strong correlations between the items on the MRF-L and the sub scales of the Competitive State Anxiety Inventory 2 that it is designed to substitute for in highly time constrained situations.

Rating Scale for Mental Effort (RSME). The RSME (Zijlstra, 1993) was used to assess mental effort. It is a one-dimensional linear scale which runs from 0 150 with zero corresponding to not at all effortful, 75 corresponding to moderately 
1 effortful, and 150 to very effortful. The scale has been reported as a valid and reliable

2 measure of mental effort (see Veltman \& Gaillard, 1996).

Visual Search. A Mobile-Eye gaze tracking system (Applied Science

4 Laboratories, Bedford, MA, USA) was used to record gaze behaviours. The Mobile-

5 Eye employs a monocular video-based system to record point of gaze in relation to a

6 head mounted scene camera. The system measures the relative position of the pupil

7 and corneal reflection at a functional rate of $50 \mathrm{~Hz}$ and has a manufacturer-reported

8 spatial accuracy of $0.5^{\circ}$ and a precision of $0.1^{\circ}$ of visual angle.

Kinematics. Two high-definition digital video cameras (Panasonic HC-V720

$\mathrm{HD}$, Berkshire, UK) sampling at $50 \mathrm{~Hz}$ were used to capture spatio-temporal

11 information from each trial. One camera recorded the full pitch from side on and was used to judge the length of delivery based on calibration information relating to each 1 $m$ interval along the pitch. A second camera side on to the pitch was centred inside a

field of view spanning from the stumps to four metres down the pitch to record the participant's movements. Two-dimensional spatial data from this second camera were reconstructed using calibration coefficients determined at the start of each session from a $4.00 \times 1.60 \mathrm{~m}$ frame. Temporal data from the two cameras were synchronised to the nearest millisecond using banks of LEDs which were visible in the field of view of both cameras.

\section{Procedure and Experimental Task}

Prior to taking part, participants underwent training in providing verbal reports using

Ericsson and Kirk's (2001) adaptation of Ericsson and Simon's (1993) original protocol. Training included instruction on thinking aloud and giving immediate retrospective verbal reports by solving a range of generic and domain-specific tasks 
1 (see Eccles, 2012). The verbal report training protocol lasted approximately 30

2 minutes. The Mobile-Eye system was then placed on the participant's head and

3 calibrated. The calibration involved the use of the bowler holding up the ball in five

4 locations around the body. Participants were informed how to use the MRF-L and

5 RSME and faced six familiarisation deliveries from the bowler. While facing these

6 familiarisation deliveries, participants were asked to practise giving retrospective

7 verbal reports. If these reports were not satisfactory (i.e. reports were summarised or

8 explained), the participant was reminded of their verbal report training and given

9 further training. Participants wore the Mobile-Eye system during familiarisation,

10 allowing them to become accustomed to batting while wearing the equipment. For the

11 experimental task, the bowler was instructed to bowl as he would in a match situation.

12 An observer, acting as an umpire positioned behind the stumps at the bowler's end,

13 immediately judged if each delivery was of full length (allowing the batsman to move

14 forward to the ball) and straight (between the line of the middle stump and the wide

15 line of the off side). Participants were unaware of the delivery inclusion criteria and

16 batted in each experimental condition until they had received 18 deliveries that were

17 judged by the observer to be full and straight. Due to the positioning of the observer

18 looking down the line of the delivery, whether it was sufficiently straight could be

19 determined during data collection. However, the length at which the ball bounced was

20 difficult to determine during data collection and was therefore quantitatively analysed

21 from the video images following data collection. From the deliveries that were judged

22 to have been straight, those deliveries that were measured to bounce between 3 and 7

23 metres from the stumps were deemed to be of full length and were used for

24 subsequent analysis (see Figure 1). All conditions for every participant contained at

25 least 15 qualifying deliveries, therefore the first 15 qualifying deliveries from each 
1 condition were used for analysis, equating to 60 trials per participant. On four random

2 occasions during each condition, participants were prompted to provide an immediate

3 retrospective verbal report of their thoughts while facing the delivery they had just

4 faced. On these trials, participants were asked to verbally indicate their MRF-L and

5 RSME scores. Participants were informed that their verbal reports should include all

6 of their thoughts from the end of the previous trial to the end of the trial being

7 reported on.

8

9

10

11

12

13

14

anxiety and the presence of situation-specific context. Anxiety was manipulated using

a combination of peer comparison, false feedback, and financial reward. Participants

Fig. 1 (a) Schematic showing experimental lay out, deliveries that bounced inside the grey box were used for analysis. (b) Example of the schematic shown to batsmen in context laden conditions. Black dots represent fielders and arrows potential scoring options. 
1 performance (by quality of bat-ball contact) and that a player of their level would be

2 expected to reach $75 \%$ good contacts. In addition, participants were informed they

3 had been paired with another player who had previously completed the task and

4 recorded above $75 \%$ good contacts, and that if they also completed the task; both

5 players would receive a $£ 10$ reward (all players were paid at the completion of the

6 session). During the testing under anxiety, players were given false feedback that they

7 were not reaching the required standard. For low-anxiety conditions, the participants

8 were reminded before the first trial and again half-way through the trials that they

$9 \quad$ were not being judged on performance.

A combination of situation-specific contextual information was used in an

attempt to manipulate cognitive load with a task representative of the performance

environment. Situation-specific context was manipulated by providing information on

field placing and game situations. Markers representing the positioning of fielders

were laid out and the participant was presented with a schematic of the field that

matched the location of the markers (this was available throughout the condition).

16 After being talked through the placing of the fielders, participants were informed that

17 the current game situation was 75 runs scored for the loss of two wickets, 15 overs

18 into a 50 over game and that they had come to the crease with a spinner bowling. An independent cricket coach regarded this contextual-information as a neutral situation

from an anxiety perspective that would not cause the participants to feel under

particularly high or low pressure. In total, participants completed four experimental

conditions (low anxiety + low context; low anxiety + high context; high anxiety + low

context; high anxiety + high context) with both manipulations being counterbalanced 


\section{Dependent Measures and Data Analysis}

\section{Performance Measures}

Muller and Abernethy's (2008) quality of bat-ball contact measure was used to assess performance outcome. An observer rated bat-ball contact as good, bad or no contact from the umpire's position. Good contact was defined as the ball making contact with the blade of the bat (not handle or gloves of batsman) and travelling in a direction that was consistent with the pre-contact plane of motion from the bat. Bad contact was defined as the ball making contact with the blade of the bat but travelling in a direction not consistent with the pre-contact plane of bat motion. No contact was defined as when the participant attempted to strike the ball but the blade of the bat made no contact with the ball. Trials in which the participant made no attempt to strike the ball (i.e., the participant deliberately left the delivery) were excluded from analyses.

\section{Eye-Movement Data}

Gaze behaviour data were coded frame-by-frame using $120 \mathrm{~ms}$ (three frames) as the minimum time required for a fixation (Panchuck \& Vickers, 2006). Gaze fixations were categorised into pre-selected locations. Fixation categories were selected based on McRobert et al. (2011) and included: ball/hand; bowling arm; nonbowling arm; head/shoulders; trunk/hips; legs; predicted ball release point; umpire; and unclassified. The height of the bowler equated to $7^{\circ}$ of visual field. Fixations were measured as staying within one cursor width, equating to $1^{\circ}$ of visual field or 28 $\mathrm{cm}$ on the bowler's body. Due to occasional loss of calibration during testing, complete eye-movement data were available for 9 of the 12 participants.

\section{Verbal Reports}



on the structure outlined by Ericsson and Simon (1993) and developed by Ward, Williams, and Ericsson (2003). The four categories were: (i) monitoring statements, recalling descriptions of current events and current actions; (ii) planning, referring to

5 potential decisions on a course of action to anticipate an outcome event; (iii)

6 predictions, referring to statements anticipating or highlighting possible future events;

7 and (iv) evaluations, referring to statements making some form of comparison,

8 assessment or appraisal of events that are situation, task, or context relevant.

\section{Kinematics}

Two-dimensional spatial kinematics of the participants were reconstructed from the video clips using Vicon Motus software (v. 9.2.0, Vicon, Oxford, UK). The type of shot chosen and specific movement characteristics for analysis were chosen based on the work of Pinder, Renshaw, and Davids (2009). Due to the representative nature of the task, the participants utilised numerous different responses to deliveries that bounced at the same point, hence using all qualifying deliveries would lead to comparing kinematics of numerous types of shot. Analyses were therefore restricted to deliveries to which the batsman executed a forward defensive shot. Across the four conditions, participants executed a total of 157 forward defensive shots. Specific spatial measures included peak height of the toe of the bat relative to the floor prior to shot execution (i.e. during the backswing), horizontal displacement of the batsman's front foot when it was on the ground and the horizontal displacement of the contact point (where the bat intercepted the ball), both measured from the stumps at the batsman's end. The difference between the horizontal positions of the front foot and the contact point was calculated to identify whether the ball was played in front or behind the front foot (a positive value represents the ball played ahead of the foot). 
1 Temporal analyses were based on the work of Pinder, Renshaw, and Davids (2009)

2 and required identification of the initiation of backswing, initiation of front foot

3 movement, front foot placement and initiation of downswing (all expressed relative to

4 the time of ball contact). The time difference between the initiation of the downswing

5 and front foot placement was also calculated. Synchronisation LED information was

6 not available for two participants so temporal kinematics were available for 10 of the

$7 \quad 12$ participants.

\section{Data Analysis}

A number of separate two-way repeated measures ANOVAs were used to analyse the effect of anxiety and situation-specific context on MRF-L scores, RSME, quality of bat-ball contact, number of fixations, number of fixation locations, fixation duration, and each spatial and temporal kinematic measure, respectively. Three-way repeated measures ANOVAs were used to analyse verbal reports and percentage viewing times. A Bonferroni adjustment was employed when multiple comparisons were being made in order to lower the significance threshold and avoid Type I errors

(McLauglin \& Sainani, 2014). Violations of sphericity were corrected for by adjusting

the degrees of freedom using the Greenhouse Geisser correction when epsilon was less than 0.75 and the Huynh-Feldt correction when greater than 0.75 (Girden, 1992).

Partial eta squared $\left(\eta_{p}^{2}\right)$ was used as a measure of effect size for all analyses.

Pearson's correlation coefficient ( $r$ ) was used to calculate the relationship between the timings of front foot placement and downswing. The alpha level $(p)$ for statistical significance was set at 0.05 .

\section{Results}

\section{The Mental Readiness Form - Likert (MRF-L)}


Our anxiety manipulation had a significant effect on participants' reported

2

$\left.4 \quad{ }_{11}=9.30, p=0.01, \eta_{p}{ }^{2}=0.46\right)$. Our anxiety manipulation had no effect on

5 participants' reported somatic anxiety or self-confidence (all $F^{\prime} s \leq 3.05, p^{\prime} s>0.05$ ).

6 Context manipulations did not affect reported levels of cognitive anxiety, somatic

7 anxiety or self-confidence (all $F^{\prime} s \leq 3.05, p^{\prime} \mathrm{s}>0.05$ ) There was no significant anxiety

$8 \times$ context interaction for cognitive anxiety, somatic anxiety or confidence (all $F^{\prime} s \leq$

$9 \quad 3.05, p$ 's $>0.05)$.

cognitive anxiety. Participants reported higher levels of cognitive anxiety under the high- $(M=3.92 \pm 1.34)$ compared with low-anxiety $(M=3.38 \pm 1.34)$ condition $\left(F_{l,}\right.$

\section{The Rating Scale for Mental Effort (RSME)}

The anxiety and context manipulations had no effect on mental effort (low $M$ $=57.92 \pm 16.76$, high $M=59.77 \pm 21.64, F_{1,11}=0.38, p=0.55, \eta_{p}{ }^{2}=0.03 ;$ low $M=$ $57.06 \pm 19.42 ;$ high $\left.M=60.64 \pm 19.16 ; F_{1,11}=2.57, p=0.14, \eta_{p}{ }^{2}=0.19\right)$

respectively. The anxiety $\times$ context interaction was not significant $\left(F_{1,11}=0.24, p=\right.$ $\left.0.63, \eta_{p}^{2}=0.02\right)$.

\section{Performance}

There was a significant effect of anxiety manipulation on the quality of batball contacts (Figure 2). Participants made a lower percentage of good contacts under the high- $(M=57.78 \pm 12.99)$ compared with low-anxiety $\left(M=70.56 \pm 15.47 ; F_{1,11}=\right.$ $\left.6.26, p=0.03, \eta_{p}{ }^{2}=0.36\right)$ condition. There were significantly more bad contacts under high- $(M=33.33 \pm 13.33)$ compared with low-anxiety $\left(M=23.06 \pm 11.29 ; F_{l}\right.$, ${ }_{11}=5.13, p=0.05, \eta_{p}{ }^{2}=0.32$ ). Anxiety had no effect on the number of times the ball was missed (low $M=6.39 \pm 6.66$, high $M=8.89 \pm 7.78 ; F_{1,11}=2.46, p=0.15, \eta_{p}{ }^{2}=$ 0.18). There was a significant effect of context manipulation on the number of times no contact was made with the ball. Participants missed the ball more with situation- 
1 specific context $(M=10.28 \pm 8.56)$ compared with the without-situation-specific

2 context condition $\left(M=5.00 \pm 4.50 ; F_{1,11}=9.48, p=0.01, \eta_{p}{ }^{2}=0.46\right)$. Context had

3 no effect on the number of good contacts (low $M=66.39 \pm 17.30$, high $M=61.94 \pm$

$413.55, F_{1,11}=2.53, p=0.14, \eta_{p}{ }^{2}=0.19$ ) or the number of bad contacts (low $M=$

$5 \quad 28.61 \pm 15.66$, high $\left.M=27.78 \pm 10.71, F_{1,11}=0.06, p=0.80, \eta_{p}{ }^{2}=0.01\right)$. There was

6 no interaction between anxiety and context on the number of $\operatorname{good}\left(F_{1,11}=0.00, p=\right.$

$\left.7 \quad 1.00, \eta_{p}{ }^{2}=0.00\right), \operatorname{bad}\left(F_{1,11}=0.19, p=0.67, \eta_{p}{ }^{2}=0.02\right)$ or no $\operatorname{contacts}\left(F_{1,11}=0.49\right.$,

$\left.8 \quad p=0.50, \eta_{p}{ }^{2}=0.04\right)$.

Fixation Duration: The average fixation duration was shorter in the high- compared with low-anxiety condition (mean duration in milliseconds; low $M=675.09 \pm 157.06$;

24 high $\left.M=520.75 \pm 86.29 ; F_{1,8}=6.84, p=0.03, \eta_{p}{ }^{2}=0.46\right)$. The context manipulation

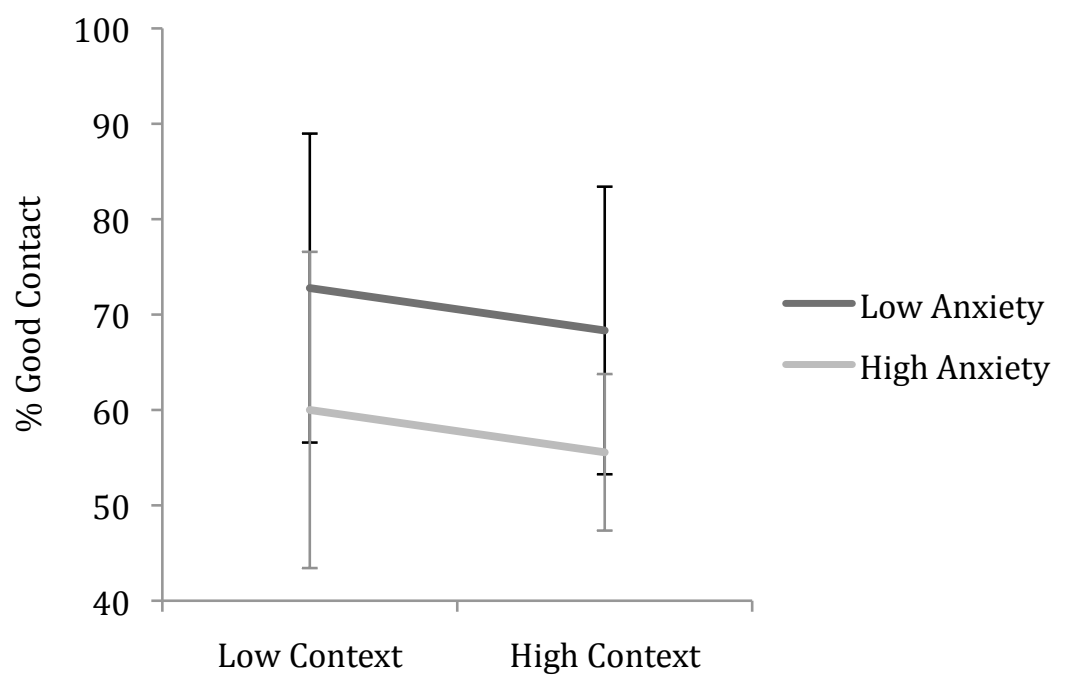

Fig 2 Percentage of deliveries with which the batsman made 'good contact' across each of the four conditions (with SD bars).

25 had no effect on fixation duration (low $M=598.31 \pm 155.04$; high $M=597.53 \pm$ 
$\left.1 \quad 141.37 ; F_{1,8}=0.00, p=0.98, \eta_{p}{ }^{2}=0.00\right)$. There was no interaction between anxiety

2 and context $\left(F_{1,8}=0.67, p=0.44, \eta_{p}{ }^{2}=0.08\right)$.

3 Number of Fixations: There was a significant effect of anxiety on the mean number

4 of fixations per second (mean fixations per second; low $M=1.51 \pm 0.29$; high $M=$

$\left.5 \quad 1.93 \pm 0.33 ; F_{1,8}=15.62, p=0.004, \eta_{p}{ }^{2}=0.66\right)$. Context had no effect on the number

6 of fixations (low $M=1.72 \pm 0.38$; high $M=1.72 \pm 0.32 ; F_{1,8}=0.00, p=0.99, \eta_{p}{ }^{2}=$

$70.000)$. There was no anxiety $\times$ context interaction $\left(F_{1,8}=1.35, p=0.28, \eta_{p}{ }^{2}=0.14\right)$.

8 Number of Fixation Locations: Participants made fixations to more locations under

9 high- compared with low-anxiety conditions (mean locations per trial; low $M=2.05 \pm$

0.34 ; high $\left.M=2.76 \pm 0.63 ; F_{1,8}=15.61, p=0.004, \eta_{p}{ }^{2}=0.66\right)$. Context did not

significantly affect the number of fixation locations (low $M=2.37 \pm 0.47$; high $M=$

$\left.2.44 \pm 0.73 ; F_{1,8}=0.138, p=0.72, \eta_{p}{ }^{2}=0.02\right)$. There was no anxiety $\times$ context

interaction $\left(F_{1,8}=0.37, p=0.56, \eta_{p}{ }^{2}=0.04\right)$.

Fixation Locations: There was a significant anxiety $\times$ location interaction on the

percentage of viewing times spent fixating different display features $\left(F_{1,8}=7.52, p=\right.$

$\left.0.01, \eta_{p}{ }^{2}=0.48\right)$. Participants fixated on ball/hand location less under high anxiety

$(51.39 \pm 6.74 \%)$ compared with low anxiety $(65.76 \pm 6.61 \%)$ and more on the

head/shoulders under high anxiety $(28.27 \pm 8.74 \%)$ compared with low anxiety $(19.68$

$\pm 7.78 \%$; see Figure 3). Post-hoc analyses revealed that participants focused

significantly longer on the ball/hand location than any other location (all $p<0.05$ )

except for the head/shoulders $(p>0.05)$. There was no context $\times$ location interaction

$\left(F_{1,8}=3.71, p=0.053, \eta_{p}{ }^{2}=0.32\right)$ or anxiety $\times$ context $\times$ location interaction $\left(F_{1,8}=\right.$

$\left.1.45, p=0.27, \eta_{p}{ }^{2}=0.15\right)$. 


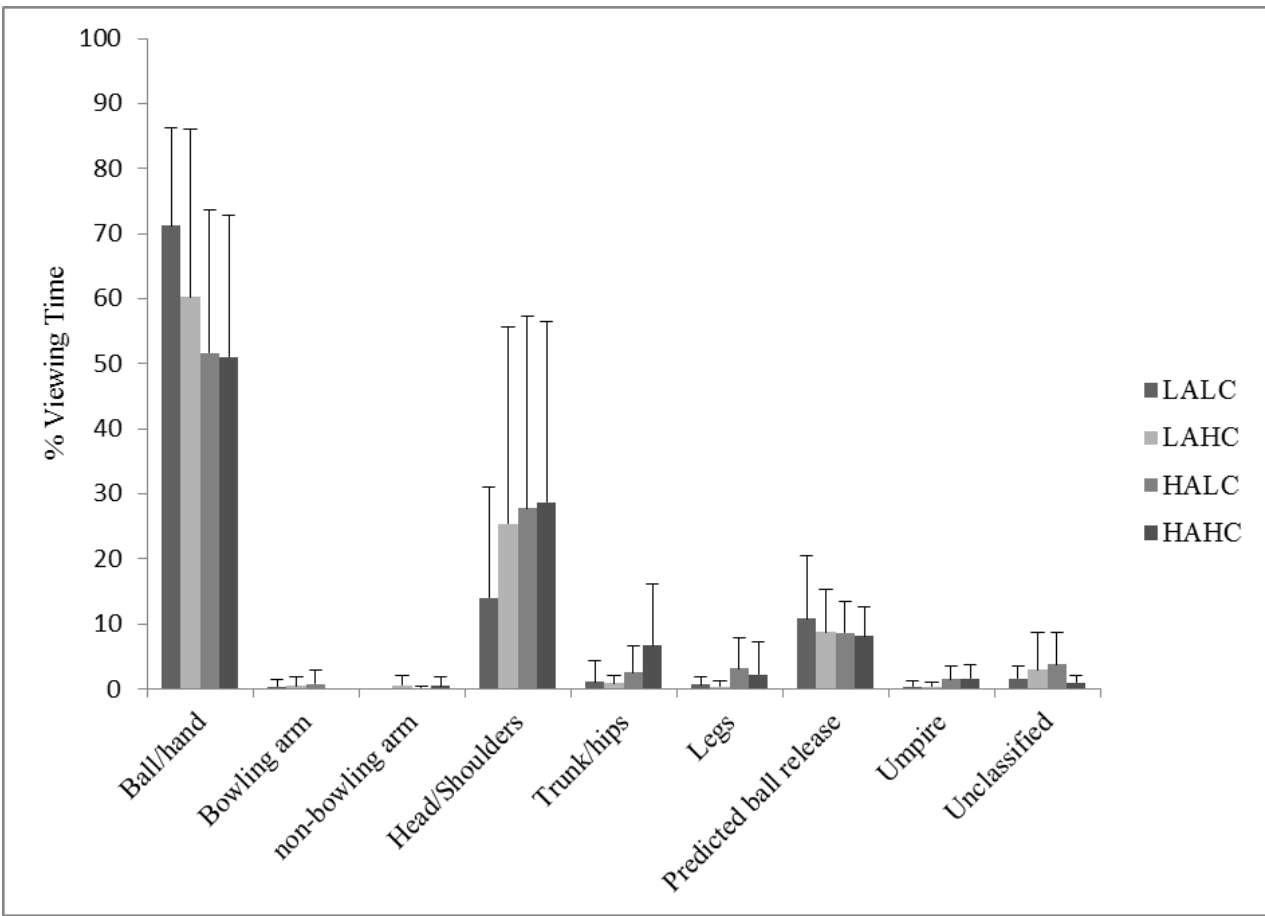

Fig. 3 Mean percentage time spent viewing each fixation location for low and high anxiety (LA, HA) and low and high context (LC, HC) (with SD bars).

\section{Verbal Reports}

There was a significant effect of statement type on number of statements made

$\left(F_{1,3}=14.45, p=0.00, \eta_{p}{ }^{2}=0.57\right)$. Post hoc analysis revealed no differences between

monitoring statements (Mean per condition $=4.69 \pm 3.54)$ and planning statements $(M$

$=4.06 \pm 2.96 ; p=0.99)$. However, both were more commonly reported than

evaluations $(M=1.23 \pm 1.28 ; p=0.01)$ and predictive statements $(M=0.23 \pm 0.66 ; p$ $=0.002$ ). Evaluation statements were reported more commonly than predictive statements $(p=0.01)$. There was no effect of the anxiety manipulation on the number of different types of verbal report statements made across conditions $\left(F_{1,11}=0.46, p=\right.$ $\left.0.42, \eta_{p}{ }^{2}=0.04\right)$. Context had no effect on type of statements verbalised $\left(F_{1,11}=0.27\right.$, $\left.p=0.91, \eta_{p}{ }^{2}=0.02\right)$. There was no anxiety $\times$ context interaction $\left(F_{1,11}=0.171, p=\right.$ 
2 Spatial Kinematics. Anxiety had no effect on any spatial kinematic measures (all $F \leq$

$3 \quad 3.05$, all $p>0.05$ ). The presence of context had no effect on peak backswing height,

4 front foot displacement or contact displacement (all $F \leq 3.05$, all $p>0.05$ ). However,

5 in context-laden conditions, participants contacted the ball significantly further behind

6 the front foot than in low-context conditions (low $M=0.07 \pm 0.15 \mathrm{~m}$; high $M=-0.10$

$\left.7 \pm 0.35 \mathrm{~m} ; F_{1,11}=5.32, p=0.04, \eta_{p}{ }^{2}=0.33\right)$. There was no anxiety $\times$ context effect on

8 any of the spatial kinematic measures (all $F \leq 3.05$, all $p>0.05$ ).

9 Temporal Kinematics Anxiety had no effect on any of the temporal kinematic measures (all $F \leq 3.05$, all $p>0.05$ ). There was no effect of context on the timing of backswing initiation, front foot movement, front foot placement or initiation of downswing and there was no interaction between anxiety and context on any temporal kinematic measures (all $F \leq 3.05$, all $p>0.05$ ). However, context did affect the correlation between the timing of the initiation of downswing and front foot placement. Under conditions with no situation-specific context, the timing of the two movements was strongly correlated $(r=0.89, p<0.01)$, meaning that if the placement of front foot occurred earlier before impact so would the initiation of the downswing. In context-laden situations, there was no significant correlation between front foot placement and downswing initiation $(r=-0.09, p=0.40)$, meaning the timing of each movement was independent of the other.

\section{Discussion}

We examined how anxiety and situation-specific context affected perceptualmotor performance through attentional, interpretational, and behavioural mechanisms using a novel in-situ task. The results supported our hypothesis based on ACT that 
1 participants would make more fixations of a shorter duration to more locations of less

2 relevance under high anxiety conditions. We had further hypothesised that, as

3 Nieuwenhuys and Oudejans (2012) suggested, decreasing efficiency of attentional

4 processes due to anxiety will negatively affect the response options available at the

5 interpretational level and lead to a decrement in the quality of batting movement

6 execution and performance. Although we found an effect of anxiety on performance

7 and attentional processes, there was no subsequent effect at interpretational or

8 behavioural levels. Furthermore, we hypothesised based on ACT, that context-laden

9 conditions would induce a higher working memory load which, when coupled with

10 high anxiety, would result in insufficient resources being available to compensate for

11 the effects of anxiety, thereby leading to a decrement in performance effectiveness.

12 Yet, although we saw no change in working memory load or anxiety due to context,

13 we still found a negative impact on performance. We hypothesised this negative effect

14 would be under-pinned by changes at all three levels, but reported that only the

situation-specific context manipulation affected mechanisms underpinning perceptual-

16 motor performance directly at the behavioural level.

Our anxiety manipulation was successful in producing a rise in cognitive

anxiety. Although the levels of anxiety experienced are likely less than those in

competition, this increase in cognitive anxiety was statistically significant and

affected both performance effectiveness (quality of bat-ball contact) and processing

efficiency (visual search behaviour). ACT predicts that extra resources from the

working memory could be used to counter negative effects of anxiety on processing

efficiency, but performance will be affected when working memory no longer has the

capacity to counteract these effects. Cocks et al. (2015) only found a decrement in 
1 information in a simulated tennis task. In our task, we observed a significant

2 decrement in batting performance (performance effectiveness) alongside a change in

3 processing efficiency due to anxiety alone; this difference could be due to the more

4 complex nature of performing an in-situ batting task relative to the simulation used by

5 Cocks et al. (2015) in which participants made responses by stepping into one of four

6 sections on a grid. Cricket batting is a highly complex interceptive action that

7 potentially already utilises a significant amount of working memory resources and,

8 therefore, prevents participants from having the capacity to compensate for the effects

9 of anxiety. No interaction was found between anxiety and context on anxiety

10 measures, which is in line with the assumption of ACT that both are acting on the

11 working memory and, therefore, operate in a cumulative rather than interactive

12 fashion.

We found a negative effect of situation-specific context on performance,

14 which was underpinned by changes in the relative timing of front foot placement and

15 downswing initiation, and positioning of the contact point in relation to the front foot.

16 These changes occurred without any corresponding change in measures of mental

17 effort or anxiety. Although these findings suggest that our attempt to manipulate

18 cognitive load with situation-specific context was not overly successful, the use of

19 situation-specific context unearthed an interesting and novel finding. The negative

20 effect of context on performance occurred without an increase in mental effort,

21 anxiety, or changes in perceptual-cognitive mechanisms. The performance decrement

22 may therefore be mediated by some other mechanism independent of working

23 memory load. Our suggestion is that performance is affected by situation-specific

24 context in a different way from anxiety. The experienced cricketers who participated

25 in this study were familiar with field placements, tactics, and game situations and, 
1 therefore, these manipulations were not sufficient to impact cognitive load. Those

2 researchers who have utilised artificial and non-representative secondary tasks to

3 target working memory load (as is typically the case in published literature testing

$4 \quad$ ACT) may not detect performance decrements that occur in representative

5 performance environments. Furthermore, findings from studies such as McRobert et

6 al. (2011) and Cocks et al. (2015) which used film-based paradigms, while measuring

7 only attentional and interpretational mechanisms, to investigate anxiety and context in

8 anticipation may not detect potentially interesting findings at the behavioural level.

9 We recorded gaze behaviour as a measure of how anxiety and situation-

10 specific context can affect mechanisms at the attentional level and as proxy for

11 processing efficiency. Participants displayed more fixations of a shorter duration to

12 more locations under high-anxiety conditions; such a visual search strategy typically

13 characterises novice or lesser skilled performers. Although gaze behaviours are task,

14 context and situation specific, it has been reported that making more fixations of a

15 shorter duration is a less efficient way of processing information due to less time

16 spent on task relevant cues and more inactive periods of information processing

17 during saccades (see Mann, Williams, Ward, \& Janelle, 2007). When considered

18 against the predictions of ACT, these less efficient visual search behaviours are most

19 likely due to a reduction in the inhibition function, which results in more time spent

20 attending to threat-related irrelevant stimuli rather than specific task-relevant stimuli.

21 Our findings support the prediction of ACT that processing efficiency decreases under 22 anxiety.

23 Our results revealed the effects of anxiety were restricted to attentional processes with no significant change in interpretational or behavioural processes (as assessed through verbal reports and kinematic analyses respectively). These 
1 observations suggest that in contrast to the predictions made by Nieuwenhuys and

2 Oudejans (2012), the effects of anxiety on the attentional, interpretational, and

3 behavioural mechanisms that are engaged during the performance and control of

4 perceptual-motor tasks may not be linear in nature. However, these findings are in

5 contrast to Whitehead, Taylor, and Polman (2016) who found an increase in

6 verbalised technical rules under anxiety, supporting execution-focused theories of

7 how anxiety can affect perceptual-motor control, as proposed in Masters and

8 Maxwell's (2008) Reinvestment Hypothesis. It is possible that, due to the significant

9 time constraints placed on participants between ball release and shot execution in a

10 cricket batting task, there is not sufficient time available to consider technical rules.

11 Furthermore, there was no evidence that changes in anxiety or context could affect the

12 use of high- or low-level of cognitive processes. It is somewhat surprising that high-

13 context conditions did not induce the use of higher-order cognitive processes such as

14 use of tactics and planning statements. However, this may be due to the nature of the

15 task, in which the batsman's selection of shots is still often dictated by where the ball

16 is delivered by the bowler under severe time constraint.

Regarding the lack of change in behavioural level measures under anxiety

18 (i.e., spatial and temporal kinematics), our findings were in contrast to those of Causer

19 et al. (2011) who reported a change in the gun kinematics of elite shotgun shooters as

20 well as in visual search behaviour under high anxiety. However, although anxiety had

21 no significant effects on behavioural measures, we did show that changes in

22 movement execution occurred due to the presence of situation-specific context.

23 Context broke the relationship between the timing of the front foot placement and

24 initiation of downswing as well as causing the ball to be contacted significantly

25 further behind the front foot. This strategy could be viewed as a less aggressive way 
1 of batting. While the anxiety negatively affected performance through changes in

2 attentional mechanisms, it had no consequent effects on movement execution. Context

3 had no effect on attentional or interpretational mechanisms, but negatively affected

4 performance separately through mechanisms directly at the behavioural level. Causer

5 et al. (2011) used competition scenarios to manipulate anxiety; it could be the case

6 that anxiety affected the attentional mechanisms but the addition of contextual

7 information in the form of a performance scenario separately impacted the gun

8 kinematics rather than it being due to anxiety. In the current paper, we have shown

9 that anxiety and context manipulations can affect individual mechanisms of

10 perceptual-motor control independently of each other, challenging the assumption that

11 there could be consequent effects from attention to behaviour in the motor control

12 process.

Although we used a relatively small sample size, which could represent a

14 potential limitation with this study, this allowed for a highly specialised population to

15 be studied at multiple levels in a representative environment and produced a number

16 of significant findings that have implications for theoretical development and applied

17 practice. First, the finding that situation-specific context did not affect mental effort

18 scores but still caused a performance decrement shows that when a task is made more complex using situation-specific information, it affects performance without impacting cognitive load. It is possible that the measure used for cognitive load was not effective in this setting and therefore no effects were found, however, if situationspecific context does not affect cognitive load, it is likely that anxiety will be the only

23 factor to contribute to working memory load. This means that findings from experimental designs utilising non-representative secondary tasks to load working memory may not be applicable to performance environments. 
Findings at the attentional level, relating to ACT, support the predictions that

2 anxiety will have a negative impact on processing efficiency and, in turn, negatively

3 affect performance effectiveness. However, our findings suggest that the load on

4 working memory may not be the only constraint on performance under anxiety, and

5 situational context should also be considered. Furthermore, these findings show that

6 changes to attentional mechanisms may not necessarily impact mechanisms at the

7 interpretational and behavioural levels. The results we have presented have increased

8 understanding of how anxiety can affect the performance of interceptive actions in-

9 situ and how situation-specific context might impact these mechanisms. This

10 development may open an interesting course of investigation into how situation-

11 specific context using representative task designs might affect performance

12 independent of anxiety.

Findings have implications for the execution of perceptual-motor skill under

14 pressure. Even the low levels of anxiety induced in this study have resulted in a significant negative effect on performance and altered the visual search strategies employed by participants. Oudejans and Pijpers (2010) suggested that training under

even relatively low to moderate levels of anxiety can help limit the negative effects of anxiety in the performance environment. This finding supports the conclusions of Headrick, Renshaw, Davids, Pinder, and Araújo (2015) and Alder, Ford, Causer and

Williams (2016) who argue for the use of emotion to create more representative learning environments and aid better transfer to performance environments. If situation-specific context information can alter performance effectiveness through mechanisms other than anxiety, this should be taken into consideration when designing future testing and training interventions. 
In summary, we have examined how situation-specific contextual information

2 and anxiety affect perceptual-motor skill at multiple levels using a novel in-situ task.

3 We have shown that anxiety affects performance by influencing mechanisms at the

4 attentional level; however, this effect occurred without influencing interpretational or

5 behavioural mechanisms. Situation-specific context also affected performance

6 through mechanisms directly at the behavioural level. These findings have

7 implications for the use of secondary tasks in anxiety research and for the design of

8 training environments to facilitate skill learning.

\section{Compliance with Ethical Standards:}

11 Conflict of interest: Oliver Runswick declares that he has no conflict of interest.

12 André Roca declares that he has no conflict of interest. Mark Williams declares that

13 he has no conflict of interest. Neil Bezodis declares that he has no conflict of interest.

14 Jamie North declares that he has no conflict of interest

16 Ethical approval: All procedures performed in studies involving human participants

17 were in accordance with the ethical standards of the institutional research committee and with the 1964 Helsinki declaration and its later amendments or comparable

19 ethical standards.

21 Informed consent: Informed consent was obtained from all individual participants 22 included in the study. 


\section{References}

2 Alder, D., Ford, P. R., Causer, J., \& Williams, A. M. (2016). The effects of high- and low-anxiety training on the anticipation judgements of elite performers. Journal of Sport and Exercise Psychology, 38, 93-104.

Broadbent, D. P., Causer, J., Williams, A. M., \& Ford, P. R. (2014). Perceptualcognitive skills training and its transfer to expert performance in the field: future research directions. European Journal of Sport Science, 4, 1-10.

Cañal-Bruland, R., \& Mann, D. L. (2015). Time to broaden the scope of research on anticipatory behaviour: a case for the role of probabilistic information. Frontiers in Psychology, 6, 1518.

Causer, J., Holmes, P. S., Smith, N. C., \& Williams, A. M. (2011). Anxiety, movement kinematics, and visual attention in elite-level performers. Emotion, $11,595-602$.

Cocks, A. J., Jackson, R. C., Bishop, D. T., \& Williams, A. M. (2015). Anxiety, anticipation and contextual information: A test of attentional control theory. Cognition and Emotion, 25, 1-12.

Corbetta, M., \& Shulman, G. L. (2002). Control of goal-directed and stimulus-driven attention in the brain. Nature Reviews Neuroscience, 3, 201-215.

Derakshan, N., \& Eysenck, M. W. (2009). Anxiety, processing efficiency, and cognitive performance: new developments from attentional control theory. European Psychologist, 14, 168-176.

Dicks, M., Button, C., \& Davids, K. (2010). Examination of gaze behaviours under in situ and video simulation task constraints reveals differences in information 
3 Eccles, D. W. (2012). Verbal reports of cognitive processes. In G. Tenenbaum, R. C.

pickup for perception and action. Attention, Perception \& Psychophysics, 72, $706-720$. Eklund, \& A. Kamata, Measurement in Sport and Exercise Psychology. Champaign, IL: Human Kinetics.

Ericsson, K. A., \& Simon, H. A. (1993). Protocol analysis: Verbal reports as data (rev. ed.). Cambridge, MA: Bradford Books/ MIT Press.

Ericsson, K. A., \& Kirk, E. (2001). Instructions for giving retrospective verbal reports. Tallahassee, FL: Florida State University.

Eysenck, M. W., \& Calvo, M. G. (1992). Anxiety and performance: The processing efficiency theory. Cognition and Emotion, 6, 409-434.

Eysenck, M. W., Derakshan, N., Santos, R., \& Calvo, M. G. (2007). Anxiety and Cognitive Performance: Attentional Control Theory. Emotion, 7, 336-353.

Girden, E. R. (1992). ANOVA: Repeated Measrues. Newbury Park, CA: Sage Headrick, J., Renshaw, I., Davids, K., Pinder, R. A., \& Araújo, D. (2015). The dynamics of expertise acquisition in sport: The role of affective learning design . Pscyhology of Sport and Exercise, 16, 83-90.

Janelle, C. M. (2002). Anxiety, arousal and visual attention: A mechanistic account of performance variability. Journal of Sports Sciences, 20, 237-251.

Krane, V. (1994). The Mental Readiness Form as a measure of competitive state anxiety. The Sport Psychologist, 8, 189-202. 
1 Mann, D. Y., Williams, A. M., Ward, P., \& Janelle, C. M. (2007). Perceptualcognitive expertise in sport: A meta-analysis. Journal of Sport \& Exercise Psychology, 29, 457-478.

Masters, R. S., \& Maxwel, J. (2008). The theory of reinvestment. International Review of Sport and Exercise Psychology, 1, 160-183.

McLaughlin, M. J., \& Sainani, K. L. (2014). Bonferroni, Holm, and Hochberg corrections: Fun names, serious changes to $p$ values. $P M \& R$, 6, 544-546.

McRobert, A. P., Ward, P., Eccles, D. W., \& Williams, A. M. (2011). The effect of manipulating context-specific information on perceptual-cognitive processes during a simulated anticipation task. British Journal of Psychology, 102, 519534.

Miyake, A., Friedman, N. P., Emerson, M. J., Witzki, A. H., Howerter, A., \& Wager, T. (2000). The unity and diversity of executive functions and their contributions to complex "frontal lobe" tasks: A latent variable analysis. Cognitive Psychology, 41, 49-100.

Muller, S., \& Abernethy, B. (2008). Validity and reliability of a simple categorical tool for the assessment of interceptive skill. Journal of Science and Medicine in Sport, 11, 549-552.

Muller, S., Brenton, J., \& Rosalie, S. M. (2015). Methodological considerations for investigating expert interceptive skill in in situ settings. Sport, Exercise and Performance Psychology, 4, 254-267. 
1 Murray, N. P., \& Jannelle, C. M. (2003). Anxiety and performance: A visual search examination of processong efficiency theory. Journal of Sport \& Exercise Psychology, 25, 171-187.

4 Nibbeling, N., Daanen, H. A., Gerritsma, R. M., Hofland, R. M., \& Oudejans, R. R. 5 (2012). Effects of anxiety on a running task with and without an aiming task. Journal of Sports Sciences, 30, 11-19.

7 Nieuwenhuys, A., \& Oudejans, R. R. (2012). Anxiety and perceptual-motor performance: toward an integrated model of concepts, mechanisms, and processes. Psychological Research, 76, 747-759.

Nieuwenhuys, A., Savelsbergh, G. J., \& Oudejans, R. R. (2012). Shoot or don't shoot? Why police officers are more inclined to shoot when they are anxious. Emotion, 12, 827-833.

Oudejans, R. R., \& Pijpers, J. R. (2010). Training with mild anxiety may prevent choking under higher levels of anxiety. Psychology of Sport and Exercise, 11, 44-50.

Oxford Dictionaries, Oxford University Press. (2016). Retrieved May 11, 2016, from $<\mathrm{http}: / /$ www.oxforddictionaries.com/definition/english/context $>$.

Panchuck, D., \& Vickers, J. N. (2006). Gaze behaviours of goal tenders under spatialtemporal constraints. Human Movement Science, 25, 733-752.

Paull, G., \& Glencross, D. (1997). Expert perception in decision making in Baseball. International Journal of Sport Pscyhology, 28, 35-56. 
1 Pijpers, J. R., Oudejans, R. R., Bakker, F. C., \& Beek, P. J. (2006). The role of anxiety in perceiving and realizing affordances. Ecological Psychology, 18, $131-161$.

4 Pinder, R. A., Davids, K., Renshaw, I., \& Araújo, D. (2011). Representative learning 5 design and functionality of research and practice in sport. Journal of Sport \& Exercise Psychology, 33, 146-155.

Pinder, R. A., Renshaw, I., \& Davids, K. (2009). Information-movement coupling in developing cricketers under changing ecological practice constraints. Human Movement Science, 28, 468-479.

Stone, J. A., Panchuck, D., Davids, K., North, J. S., \& Maynard, I. (2014). Integrating advanced visual information with ball projection technology constrains dynamic interceptive actions. Procdedia Engineering, 72, 156-161.

Veltman, J. A., \& Gaillard, A. W. (1996). Physiological indicies of workload in a simulated flight task. Biological Psychology, 42, 323-342.

Ward, P., Williams, A. M., \& Ericsson, K. A. (2003). Underlying mechanisms of perceptual-cognitive expertise in soccer. Journal of Sport \& Exercise Psychology, 25, S136.

Whitehead, A. E., Taylor, J. A., \& Polman, R. C. (2016). Evidence for skill level differences in the thought processes of golfers during high and low pressure siutuations. Frontiers in Psychology, 6, 1974.

Wilson, M., Smith, N. C., \& Holmes, P. S. (2007). The role of effort in influenceing the effect of anxiety on performance: Testing the conflicting predicitons of 
processing effiiciency theory and the conscious processing hypothesis. British Journal of Pschology, 98, 411-428.

3 Wilson, M., Vine, S. J., \& Wood, G. (2009). The influence of anxiety on visual attentional control in basketball free throw shooting. Journal of Sport \& Exercise Psychology, 31, 152-468.

6 Zijlstra, F. R. (1993). Efficiency in work behaviour; A design approach for modern tools. Delft, Netherlands: Delft University Press. 